Acta Crystallographica Section E

Structure Reports

Online

ISSN 1600-5368

\section{5-Bromo-2,7-dimethyl-3-(4-methyl- phenylsulfinyl)-1-benzofuran}

\section{Hong Dae Choi, ${ }^{\text {a }}$ Pil Ja Seo ${ }^{a}$ and Uk Lee ${ }^{b *}$}

aDepartment of Chemistry, Dongeui University, San 24 Kaya-dong, Busanjin-gu, Busan 614-714, Republic of Korea, and ${ }^{\mathbf{b}}$ Department of Chemistry, Pukyong

National University, 599-1 Daeyeon 3-dong, Nam-gu, Busan 608-737, Republic of Korea

Correspondence e-mail: uklee@pknu.ac.kr

Received 28 March 2013; accepted 9 April 2013

Key indicators: single-crystal X-ray study; $T=173 \mathrm{~K}$; mean $\sigma(\mathrm{C}-\mathrm{C})=0.005 \AA$; $R$ factor $=0.045 ; w R$ factor $=0.089 ;$ data-to-parameter ratio $=17.3$.

In the title compound, $\mathrm{C}_{17} \mathrm{H}_{15} \mathrm{BrO}_{2} \mathrm{~S}$, the 4-methylbenzene ring makes a dihedral angle of $89.01(7)^{\circ}$ with the mean plane [r.m.s. deviation $=0.013(2) \AA]$ of the benzofuran fragment. In the crystal, molecules are linked into supramolecular layers that stack along [001] by weak $\mathrm{C}-\mathrm{H} \cdots \mathrm{O}, \mathrm{C}-\mathrm{H} \cdots \pi$ and $\mathrm{C}-$ S. $\cdot \pi[3.364(2) \AA]$ interactions.

\section{Related literature}

For background information and the crystal structures of related compounds, see: Choi et al. $(2011 a, b)$.

\section{Experimental}

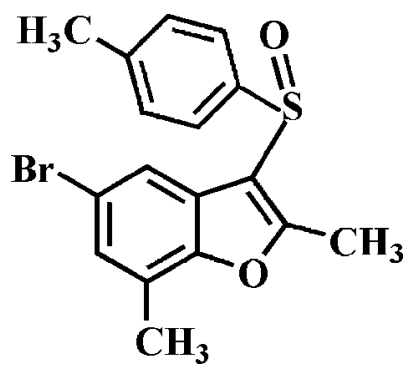

Crystal data

$\mathrm{C}_{17} \mathrm{H}_{15} \mathrm{BrO}_{2} \mathrm{~S}$

$M_{r}=363.26$

Triclinic, $P \overline{1}$

$a=6.1794$ (6) § $\gamma=85.471(6)^{\circ}$

$V=763.67(13) \AA^{3}$

$Z=2$

Mo $K \alpha$ radiation

Data collection

Bruker SMART APEXII CCD diffractometer

Absorption correction: multi-scan (SADABS; Bruker, 2009)

$T_{\min }=0.640, T_{\max }=0.746$

Refinement

$R\left[F^{2}>2 \sigma\left(F^{2}\right)\right]=0.045$

$w R\left(F^{2}\right)=0.089$

$S=1.04$

3331 reflections

Table 1

Hydrogen-bond geometry $\left(\AA,{ }^{\circ}\right)$.

$\mathrm{Cg}$ is the centroid of the C11-C16 4-methylphenyl ring.

\begin{tabular}{lllll}
\hline$D-\mathrm{H} \cdots A$ & $D-\mathrm{H}$ & $\mathrm{H} \cdots A$ & $D \cdots A$ & $D-\mathrm{H} \cdots A$ \\
\hline $\mathrm{C} 12-\mathrm{H} 12 \cdots \mathrm{O} 2{ }^{\mathrm{i}}$ & 0.95 & 2.58 & $3.338(4)$ & 137 \\
$\mathrm{C} 17-\mathrm{H} 17 C \cdots C g^{\mathrm{ii}}$ & 0.98 & 2.77 & $3.739(4)$ & 169 \\
\hline
\end{tabular}

Symmetry codes: (i) $-x+2,-y+1,-z+1$; (ii) $-x+1,-y+2,-z+1$.

Data collection: APEX2 (Bruker, 2009); cell refinement: SAINT (Bruker, 2009); data reduction: $S A I N T$; program(s) used to solve structure: SHELXS97 (Sheldrick, 2008); program(s) used to refine structure: SHELXL97 (Sheldrick, 2008); molecular graphics: ORTEP-3 for Windows (Farrugia, 2012) and DIAMOND (Brandenburg, 1998); software used to prepare material for publication: SHELXL97.

This work was supported by the Blue-Bio Industry Regional Innovation Center (RIC08-06-07) at Dongeui University as an RIC program under the Ministry of Knowledge Economy and Busan city.

Supplementary data and figures for this paper are available from the IUCr electronic archives (Reference: TK5212).

\section{References}

Brandenburg, K. (1998). DIAMOND. Crystal Impact GbR, Bonn, Germany. Bruker (2009). $A P E X 2, S A D A B S$ and $S A I N T$. Bruker AXS Inc., Madison, Wisconsin, USA.

Choi, H. D., Seo, P. J., Son, B. W. \& Lee, U. (2011a). Acta Cryst. E67, o351.

Choi, H. D., Seo, P. J., Son, B. W. \& Lee, U. (2011b). Acta Cryst. E67, o1039.

Farrugia, L. J. (2012). J. Appl. Cryst. 45, 849-854.

Sheldrick, G. M. (2008). Acta Cryst. A64, 112-122. 


\section{supporting information}

Acta Cryst. (2013). E69, o720 [https://doi.org/10.1107/S1600536813009756]

\section{5-Bromo-2,7-dimethyl-3-(4-methylphenylsulfinyl)-1-benzofuran}

\section{Hong Dae Choi, Pil Ja Seo and Uk Lee}

\section{S1. Comment}

As a part of our continuing study of 5-bromo-2,7-dimethyl-1-benzofuran derivatives containing 4-fluorophenylsulfinyl (Choi et al., 2011a) and 4-cyclohexylsulfinyl (Choi et al., 2011b) substituents in the 3-position, we report herein the crystal structure of the title compound.

In the title molecule (Fig. 1), the benzofuran unit is essentially planar, with a mean deviation of 0.013 (2) $\AA$ from the least-squares plane defined by the nine constituent atoms. The dihedral angle between the 4-methylbenzene ring and the mean plane of the benzofuran ring is $89.01(7)^{\circ}$. In the crystal structure (Fig. 2), molecules are connected by weak C$\mathrm{H} \cdots \mathrm{O}$ and $\mathrm{C}-\mathrm{H} \cdots \pi$ interactions (Table $1, \mathrm{Cg}$ is the centroid of the C11-C16 4-methylphenyl ring), and by intermolecular $\mathrm{C}-\mathrm{S} \cdots \pi$ interactions between the sulfur atom and the 4-methylphenyl ring of an adjacent molecule, with a S1 $\cdots \mathrm{Cg}^{\mathrm{iii}}$ being 3.364 (2) ^.

\section{S2. Experimental}

3-Chloroperoxybenzoic acid (77\%, $224 \mathrm{mg}, 1.0 \mathrm{mmol})$ was added in small portions to a stirred solution of 5-bromo-2,7dimethyl-3-(4-methylphenylsulfanyl)-1-benzofuran $(302 \mathrm{mg}, 0.9 \mathrm{mmol})$ in dichloromethane $(30 \mathrm{~mL})$ at $273 \mathrm{~K}$. After being stirred at room temperature for $4 \mathrm{~h}$, the mixture was washed with saturated sodium bicarbonate solution and the organic layer was separated, dried over magnesium sulfate, filtered and concentrated at reduced pressure. The residue was purified by column chromatography (hexane-ethyl acetate, 1:1 v/v) to afford the title compound as a colourless solid [yield 79\%, M.pt: 406-407 K; $R_{\mathrm{f}}=0.63$ (hexane-ethyl acetate, 1:1 v/v)]. Single crystals suitable for X-ray diffraction were prepared by slow evaporation of its acetone solution held at room temperature.

\section{S3. Refinement}

All $\mathrm{H}$ atoms were positioned geometrically and refined using a riding model, with $\mathrm{C}-\mathrm{H}=0.95 \AA$ for aryl and $0.98 \AA$ for methyl $\mathrm{H}$ atoms. $U_{\text {iso }}(\mathrm{H})=1.2 U_{\mathrm{eq}}(\mathrm{C})$ for aryl and $1.5 U_{\mathrm{eq}}(\mathrm{C})$ for methyl $\mathrm{H}$ atoms. The positions of methyl hydrogens were optimized rotationally. 


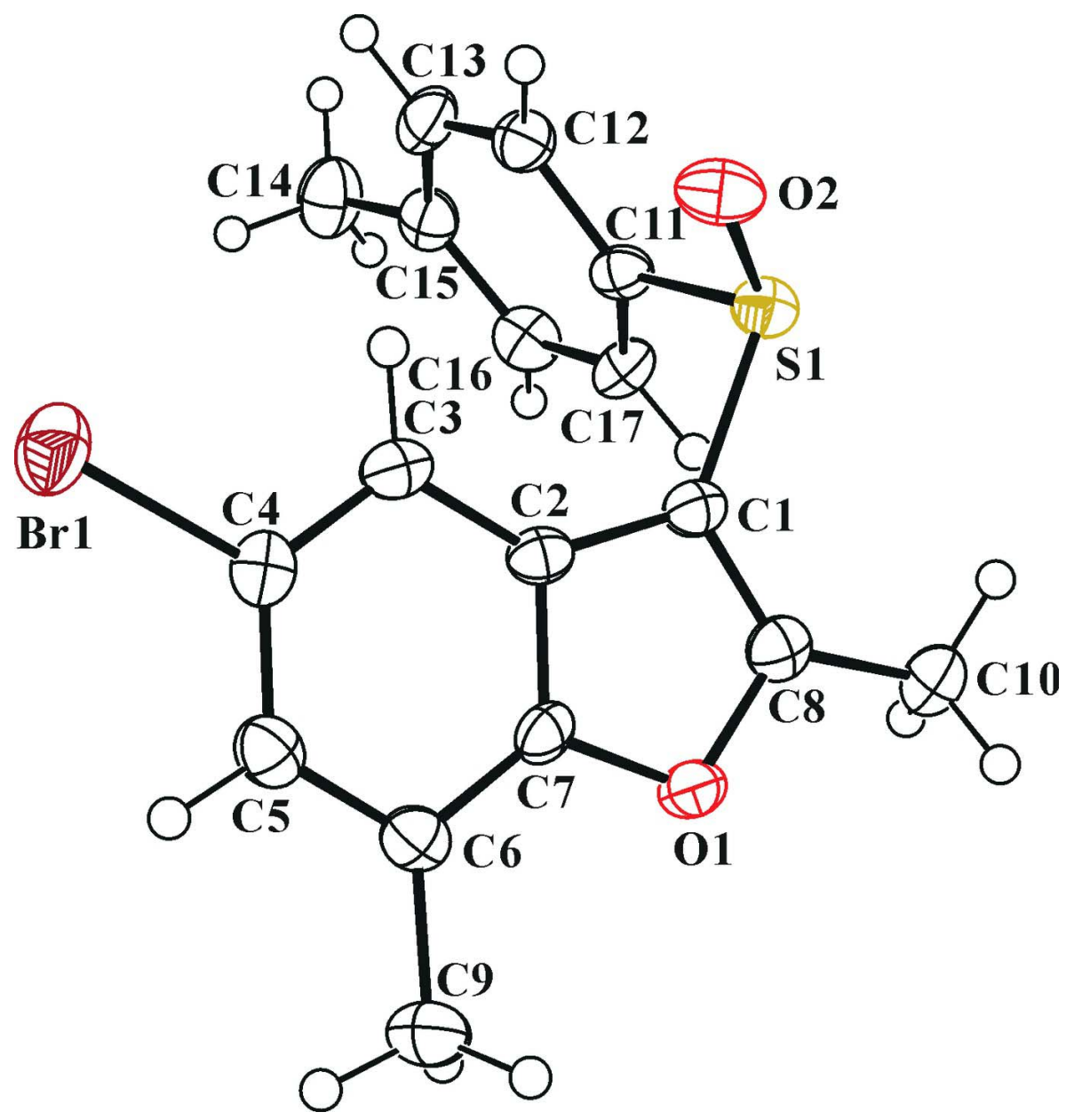

Figure 1

The molecular structure of the title compound with the atom numbering scheme. Displacement ellipsoids are drawn at the $50 \%$ probability level. $\mathrm{H}$ atoms are presented as small spheres of arbitrary radius. 

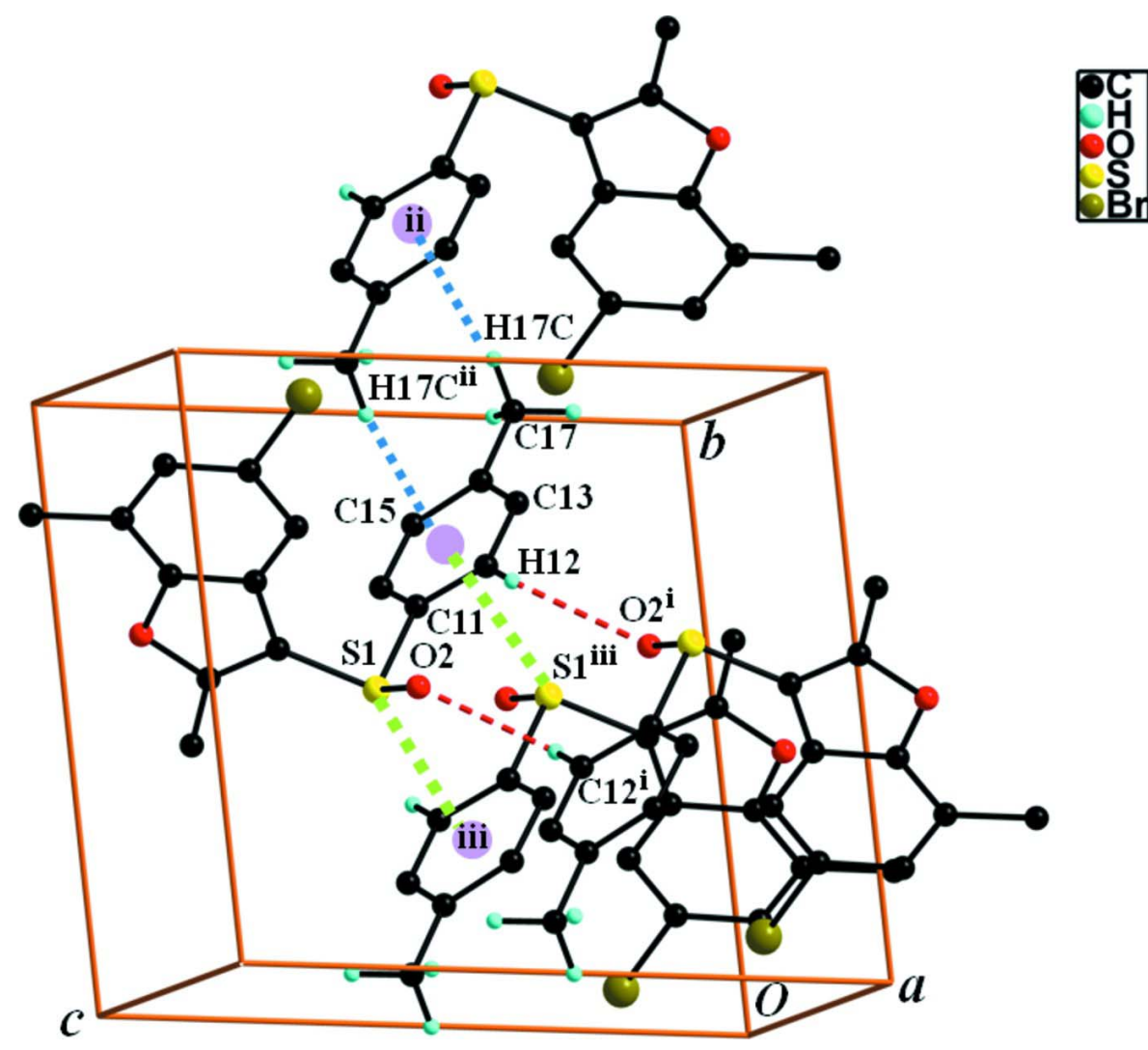

Figure 2

A view of the $\mathrm{C}-\mathrm{H} \cdots \mathrm{O}, \mathrm{C}-\mathrm{H} \cdots \pi$ and $\mathrm{C}-\mathrm{S} \cdots \pi$ interactions (dotted lines) in the crystal structure of the title compound. $\mathrm{H}$ atoms non-participating in hydrogen-bonding were omitted for clarity. [Symmetry codes: (i) $-x+2,-y+1,-z+1$; (ii) $x+1,-y+2,-z+1 ;$ (iii) $-x+1,-y+1,-z+1$.]

5-Bromo-2,7-dimethyl-3-(4-methylphenylsulfinyl)-1-benzofuran

Crystal data

$\mathrm{C}_{17} \mathrm{H}_{15} \mathrm{BrO}_{2} \mathrm{~S}$

$M_{r}=363.26$

Triclinic, $P \overline{1}$

Hall symbol: -P 1

$a=6.1794$ (6) $\AA$

$b=10.057(1) \AA$

$c=12.5793(12) \AA$

$\alpha=84.072(6)^{\circ}$

$\beta=79.738(6)^{\circ}$

$\gamma=85.471(6)^{\circ}$

$V=763.67(13) \AA^{3}$

\section{Data collection}

\section{Bruker SMART APEXII CCD}

diffractometer

Radiation source: rotating anode

Graphite multilayer monochromator

Detector resolution: 10.0 pixels $\mathrm{mm}^{-1}$

$\varphi$ and $\omega$ scans
$Z=2$

$F(000)=368$

$D_{\mathrm{x}}=1.580 \mathrm{Mg} \mathrm{m}^{-3}$

Melting point $=406-407 \mathrm{~K}$

Mo $K \alpha$ radiation, $\lambda=0.71073 \AA$

Cell parameters from 3261 reflections

$\theta=2.4-26.4^{\circ}$

$\mu=2.83 \mathrm{~mm}^{-1}$

$T=173 \mathrm{~K}$

Block, colourless

$0.22 \times 0.13 \times 0.12 \mathrm{~mm}$

Absorption correction: multi-scan

(SADABS; Bruker, 2009)

$T_{\min }=0.640, T_{\max }=0.746$

13108 measured reflections

3331 independent reflections

2180 reflections with $I>2 \sigma(I)$ 


$$
\begin{aligned}
& R_{\text {int }}=0.075 \\
& \theta_{\max }=27.0^{\circ}, \theta_{\min }=2.0^{\circ} \\
& h=-7 \rightarrow 7
\end{aligned}
$$

\section{Refinement}

\section{Refinement on $F^{2}$}

Least-squares matrix: full

$R\left[F^{2}>2 \sigma\left(F^{2}\right)\right]=0.045$

$w R\left(F^{2}\right)=0.089$

$S=1.04$

3331 reflections

193 parameters

0 restraints

Primary atom site location: structure-invariant direct methods $k=-12 \rightarrow 12$

$l=-16 \rightarrow 15$

Secondary atom site location: difference Fourier map

Hydrogen site location: difference Fourier map

$\mathrm{H}$-atom parameters constrained

$w=1 /\left[\sigma^{2}\left(F_{\mathrm{o}}^{2}\right)+(0.0171 P)^{2}+0.341 P\right]$

where $P=\left(F_{\mathrm{o}}^{2}+2 F_{\mathrm{c}}^{2}\right) / 3$

$(\Delta / \sigma)_{\max }=0.001$

$\Delta \rho_{\max }=0.41$ e $\AA^{-3}$

$\Delta \rho_{\min }=-0.52$ e $\AA^{-3}$

\section{Special details}

Geometry. All esds (except the esd in the dihedral angle between two 1.s. planes) are estimated using the full covariance matrix. The cell esds are taken into account individually in the estimation of esds in distances, angles and torsion angles; correlations between esds in cell parameters are only used when they are defined by crystal symmetry. An approximate (isotropic) treatment of cell esds is used for estimating esds involving l.s. planes.

Refinement. Refinement of $\mathrm{F}^{2}$ against ALL reflections. The weighted R-factor $\mathrm{wR}$ and goodness of fit $\mathrm{S}$ are based on $\mathrm{F}^{2}$, conventional R-factors $R$ are based on $F$, with $F$ set to zero for negative $F^{2}$. The threshold expression of $F^{2}>2$ sigma $\left(\mathrm{F}^{2}\right)$ is used only for calculating R-factors (gt) etc. and is not relevant to the choice of reflections for refinement. R-factors based

\begin{tabular}{|c|c|c|c|c|}
\hline & $x$ & $y$ & $z$ & $U_{\text {iso }} * / U_{\text {eq }}$ \\
\hline $\mathrm{Br} 1$ & $1.08078(6)$ & $0.92372(4)$ & $0.82890(3)$ & $0.04049(15)$ \\
\hline $\mathrm{S} 1$ & $0.60771(14)$ & $0.49101(9)$ & $0.65693(6)$ & $0.0281(2)$ \\
\hline O1 & $0.2942(3)$ & 0.5989 (2) & $0.93938(16)$ & $0.0285(6)$ \\
\hline $\mathrm{O} 2$ & 0.8519 & $0.4747(2)$ & $0.64788(17)$ & $0.0355(6)$ \\
\hline $\mathrm{C} 1$ & $0.5062(5)$ & $0.5624(3)$ & $0.7794(2)$ & $0.0243(8)$ \\
\hline $\mathrm{C} 2$ & $0.6048(5)$ & 0.6623 & $0.8261(2)$ & $0.0237(8)$ \\
\hline $\mathrm{C} 3$ & $0.7900(5)$ & $0.7364(3)$ & $0.7958(3)$ & $0.0273(8)$ \\
\hline $\mathrm{H} 3$ & 0.8874 & 0.7271 & 0.7291 & $0.033^{*}$ \\
\hline $\mathrm{C} 4$ & $0.8251(5)$ & 0.8235 & $0.8669(3)$ & $0.0284(8)$ \\
\hline $\mathrm{C} 5$ & $0.6844(5)$ & 0.8414 & $0.9642(3)$ & $0.0303(9)$ \\
\hline H5 & 0.7164 & 0.9043 & 1.0098 & $0.036 *$ \\
\hline C6 & $0.4982(5)$ & 0.7692 & $0.9961(2)$ & $0.0273(8)$ \\
\hline $\mathrm{C} 7$ & $0.4681(5)$ & 0.6809 & $0.9246(3)$ & $0.0241(8)$ \\
\hline $\mathrm{C} 8$ & $0.3243(5)$ & 0.5271 & $0.8496(3)$ & $0.0271(8)$ \\
\hline C9 & $0.3398(6)$ & $0.7855(4)$ & $1.1002(3)$ & $0.0399(10)$ \\
\hline H9A & 0.1935 & 0.8152 & 1.0838 & $0.060^{*}$ \\
\hline H9B & 0.3911 & 0.8522 & 1.1399 & $0.060 *$ \\
\hline $\mathrm{H} 9 \mathrm{C}$ & 0.3315 & 0.6995 & 1.1447 & $0.060 *$ \\
\hline C10 & $0.1534(6)$ & $0.4332(3)$ & $0.8474(3)$ & $0.0335(9)$ \\
\hline $\mathrm{H} 10 \mathrm{~A}$ & 0.0188 & 0.4838 & 0.8317 & $0.050 *$ \\
\hline $\mathrm{H} 10 \mathrm{~B}$ & 0.1218 & 0.3813 & 0.9181 & $0.050^{*}$ \\
\hline $\mathrm{H} 10 \mathrm{C}$ & 0.2062 & 0.3723 & 0.7911 & $0.050^{*}$ \\
\hline
\end{tabular}
on $\mathrm{F}^{2}$ are statistically about twice as large as those based on F, and R- factors based on ALL data will be even larger.

Fractional atomic coordinates and isotropic or equivalent isotropic displacement parameters $\left(\AA^{2}\right)$ 


\begin{tabular}{lllll} 
C11 & $0.5490(5)$ & $0.6350(3)$ & $0.5679(2)$ & $0.0247(8)$ \\
C12 & $0.7171(5)$ & $0.6888(3)$ & $0.4924(3)$ & $0.0290(8)$ \\
H12 & 0.8642 & 0.6515 & 0.4883 & $0.035^{*}$ \\
C13 & $0.6670(6)$ & $0.7983(4)$ & $0.4229(3)$ & $0.0340(9)$ \\
H13 & 0.7823 & 0.8369 & 0.3716 & $0.041^{*}$ \\
C14 & $0.4543(6)$ & $0.8527(3)$ & $0.4259(3)$ & $0.0304(8)$ \\
C15 & $0.2879(6)$ & $0.7931(4)$ & $0.4998(3)$ & $0.0326(9)$ \\
H15 & 0.1399 & 0.8279 & 0.5020 & $0.039^{*}$ \\
C16 & $0.3330(5)$ & $0.6843(3)$ & $0.5699(3)$ & $0.0303(8)$ \\
H16 & 0.2170 & 0.6435 & 0.6192 & $0.036^{*}$ \\
C17 & $0.4004(6)$ & $0.9735(4)$ & $0.3523(3)$ & $0.0432(10)$ \\
H17A & 0.2433 & 0.9781 & 0.3477 & $0.065^{*}$ \\
H17B & 0.4880 & 0.9666 & 0.2798 & $0.065^{*}$ \\
H17C & 0.4348 & 1.0546 & 0.3814 & $0.065^{*}$ \\
\hline
\end{tabular}

Atomic displacement parameters $\left(\AA^{2}\right)$

\begin{tabular}{lllllll}
\hline & $U^{11}$ & $U^{22}$ & $U^{33}$ & $U^{12}$ & $U^{13}$ & $U^{23}$ \\
\hline Br1 & $0.0302(2)$ & $0.0392(3)$ & $0.0535(3)$ & $-0.00854(17)$ & $-0.00904(17)$ & $-0.00239(19)$ \\
S1 & $0.0288(5)$ & $0.0298(6)$ & $0.0253(5)$ & $-0.0003(4)$ & $-0.0035(4)$ & $-0.0036(4)$ \\
O1 & $0.0286(14)$ & $0.0304(15)$ & $0.0237(13)$ & $-0.0015(11)$ & $0.0017(10)$ & $-0.0003(11)$ \\
O2 & $0.0250(13)$ & $0.0460(17)$ & $0.0343(14)$ & $0.0097(11)$ & $-0.0052(10)$ & $-0.0069(12)$ \\
C1 & $0.0237(19)$ & $0.026(2)$ & $0.0219(18)$ & $0.0001(15)$ & $-0.0024(14)$ & $0.0001(16)$ \\
C2 & $0.0204(18)$ & $0.027(2)$ & $0.0226(18)$ & $0.0015(15)$ & $-0.0032(14)$ & $-0.0007(16)$ \\
C3 & $0.0243(19)$ & $0.031(2)$ & $0.0237(18)$ & $0.0013(16)$ & $-0.0006(14)$ & $0.0009(17)$ \\
C4 & $0.026(2)$ & $0.026(2)$ & $0.034(2)$ & $-0.0021(16)$ & $-0.0092(16)$ & $0.0000(17)$ \\
C5 & $0.036(2)$ & $0.028(2)$ & $0.028(2)$ & $0.0004(17)$ & $-0.0082(16)$ & $-0.0043(17)$ \\
C6 & $0.035(2)$ & $0.026(2)$ & $0.0211(18)$ & $0.0019(17)$ & $-0.0076(15)$ & $0.0001(16)$ \\
C7 & $0.0242(19)$ & $0.021(2)$ & $0.0242(19)$ & $-0.0022(16)$ & $-0.0014(14)$ & $0.0050(16)$ \\
C8 & $0.028(2)$ & $0.026(2)$ & $0.0256(19)$ & $0.0013(16)$ & $-0.0066(15)$ & $0.0030(17)$ \\
C9 & $0.048(2)$ & $0.043(3)$ & $0.027(2)$ & $-0.0022(19)$ & $0.0015(17)$ & $-0.0063(18)$ \\
C10 & $0.032(2)$ & $0.033(2)$ & $0.035(2)$ & $-0.0082(17)$ & $-0.0044(15)$ & $0.0015(18)$ \\
C11 & $0.0236(19)$ & $0.031(2)$ & $0.0201(17)$ & $-0.0027(16)$ & $-0.0042(14)$ & $-0.0040(16)$ \\
C12 & $0.0238(19)$ & $0.033(2)$ & $0.031(2)$ & $-0.0040(16)$ & $-0.0046(15)$ & $-0.0064(17)$ \\
C13 & $0.031(2)$ & $0.037(2)$ & $0.034(2)$ & $-0.0136(18)$ & $-0.0034(16)$ & $0.0002(19)$ \\
C14 & $0.036(2)$ & $0.032(2)$ & $0.0258(19)$ & $-0.0061(17)$ & $-0.0085(16)$ & $-0.0029(17)$ \\
C15 & $0.028(2)$ & $0.036(2)$ & $0.036(2)$ & $0.0038(17)$ & $-0.0113(16)$ & $-0.0051(18)$ \\
C16 & $0.025(2)$ & $0.035(2)$ & $0.0296(19)$ & $-0.0075(16)$ & $-0.0008(15)$ & $0.0011(17)$ \\
C17 & $0.052(3)$ & $0.039(3)$ & $0.041(2)$ & $-0.0099(19)$ & $-0.0174(19)$ & $0.0050(19)$ \\
& & & & & &
\end{tabular}

Geometric parameters $\left(\AA,{ }^{o}\right)$

\begin{tabular}{llll}
\hline $\mathrm{Br} 1-\mathrm{C} 4$ & $1.904(3)$ & $\mathrm{C} 9-\mathrm{H} 9 \mathrm{~B}$ & 0.9800 \\
$\mathrm{~S} 1-\mathrm{O} 2$ & $1.490(2)$ & $\mathrm{C} 9-\mathrm{H} 9 \mathrm{C}$ & 0.9800 \\
$\mathrm{~S} 1-\mathrm{C} 1$ & $1.760(3)$ & $\mathrm{C} 10-\mathrm{H} 10 \mathrm{~A}$ & 0.9800 \\
$\mathrm{~S} 1-\mathrm{C} 11$ & $1.792(3)$ & $\mathrm{C} 10-\mathrm{H} 10 \mathrm{~B}$ & 0.9800 \\
$\mathrm{O} 1-\mathrm{C} 8$ & $1.380(4)$ & $\mathrm{C} 10-\mathrm{H} 10 \mathrm{C}$ & 0.9800 \\
$\mathrm{O} 1-\mathrm{C} 7$ & $1.381(4)$ & $\mathrm{C} 11-\mathrm{C} 12$ & $1.380(4)$
\end{tabular}




\begin{tabular}{|c|c|c|c|}
\hline $\mathrm{C} 1-\mathrm{C} 8$ & $1.347(4)$ & $\mathrm{C} 11-\mathrm{C} 16$ & $1.383(4)$ \\
\hline $\mathrm{C} 1-\mathrm{C} 2$ & $1.439(4)$ & $\mathrm{C} 12-\mathrm{C} 13$ & $1.385(4)$ \\
\hline $\mathrm{C} 2-\mathrm{C} 3$ & $1.390(4)$ & $\mathrm{C} 12-\mathrm{H} 12$ & 0.9500 \\
\hline $\mathrm{C} 2-\mathrm{C} 7$ & $1.390(4)$ & $\mathrm{C} 13-\mathrm{C} 14$ & $1.379(4)$ \\
\hline $\mathrm{C} 3-\mathrm{C} 4$ & $1.369(4)$ & $\mathrm{C} 13-\mathrm{H} 13$ & 0.9500 \\
\hline $\mathrm{C} 3-\mathrm{H} 3$ & 0.9500 & $\mathrm{C} 14-\mathrm{C} 15$ & $1.387(5)$ \\
\hline $\mathrm{C} 4-\mathrm{C} 5$ & $1.389(5)$ & $\mathrm{C} 14-\mathrm{C} 17$ & $1.503(5)$ \\
\hline $\mathrm{C} 5-\mathrm{C} 6$ & $1.385(4)$ & $\mathrm{C} 15-\mathrm{C} 16$ & $1.375(4)$ \\
\hline $\mathrm{C} 5-\mathrm{H} 5$ & 0.9500 & $\mathrm{C} 15-\mathrm{H} 15$ & 0.9500 \\
\hline $\mathrm{C} 6-\mathrm{C} 7$ & $1.372(4)$ & $\mathrm{C} 16-\mathrm{H} 16$ & 0.9500 \\
\hline $\mathrm{C} 6-\mathrm{C} 9$ & $1.504(4)$ & C17-H17A & 0.9800 \\
\hline $\mathrm{C} 8-\mathrm{C} 10$ & $1.477(5)$ & C17-H17B & 0.9800 \\
\hline $\mathrm{C} 9-\mathrm{H} 9 \mathrm{~A}$ & 0.9800 & $\mathrm{C} 17-\mathrm{H} 17 \mathrm{C}$ & 0.9800 \\
\hline $\mathrm{O} 2-\mathrm{S} 1-\mathrm{C} 1$ & $106.70(14)$ & $\mathrm{H} 9 \mathrm{~A}-\mathrm{C} 9-\mathrm{H} 9 \mathrm{C}$ & 109.5 \\
\hline $\mathrm{O} 2-\mathrm{S} 1-\mathrm{C} 11$ & $106.82(14)$ & $\mathrm{H} 9 \mathrm{~B}-\mathrm{C} 9-\mathrm{H} 9 \mathrm{C}$ & 109.5 \\
\hline $\mathrm{C} 1-\mathrm{S} 1-\mathrm{C} 11$ & $96.87(15)$ & $\mathrm{C} 8-\mathrm{C} 10-\mathrm{H} 10 \mathrm{~A}$ & 109.5 \\
\hline $\mathrm{C} 8-\mathrm{O} 1-\mathrm{C} 7$ & $106.4(2)$ & $\mathrm{C} 8-\mathrm{C} 10-\mathrm{H} 10 \mathrm{~B}$ & 109.5 \\
\hline $\mathrm{C} 8-\mathrm{C} 1-\mathrm{C} 2$ & $107.4(3)$ & $\mathrm{H} 10 \mathrm{~A}-\mathrm{C} 10-\mathrm{H} 10 \mathrm{~B}$ & 109.5 \\
\hline $\mathrm{C} 8-\mathrm{C} 1-\mathrm{S} 1$ & $125.2(3)$ & $\mathrm{C} 8-\mathrm{C} 10-\mathrm{H} 10 \mathrm{C}$ & 109.5 \\
\hline $\mathrm{C} 2-\mathrm{C} 1-\mathrm{S} 1$ & $127.3(2)$ & $\mathrm{H} 10 \mathrm{~A}-\mathrm{C} 10-\mathrm{H} 10 \mathrm{C}$ & 109.5 \\
\hline $\mathrm{C} 3-\mathrm{C} 2-\mathrm{C} 7$ & $118.9(3)$ & $\mathrm{H} 10 \mathrm{~B}-\mathrm{C} 10-\mathrm{H} 10 \mathrm{C}$ & 109.5 \\
\hline $\mathrm{C} 3-\mathrm{C} 2-\mathrm{C} 1$ & $135.8(3)$ & $\mathrm{C} 12-\mathrm{C} 11-\mathrm{C} 16$ & $120.7(3)$ \\
\hline $\mathrm{C} 7-\mathrm{C} 2-\mathrm{C} 1$ & $105.3(3)$ & $\mathrm{C} 12-\mathrm{C} 11-\mathrm{S} 1$ & $119.6(2)$ \\
\hline $\mathrm{C} 4-\mathrm{C} 3-\mathrm{C} 2$ & $116.7(3)$ & $\mathrm{C} 16-\mathrm{C} 11-\mathrm{S} 1$ & $119.4(2)$ \\
\hline $\mathrm{C} 4-\mathrm{C} 3-\mathrm{H} 3$ & 121.7 & $\mathrm{C} 11-\mathrm{C} 12-\mathrm{C} 13$ & $118.6(3)$ \\
\hline $\mathrm{C} 2-\mathrm{C} 3-\mathrm{H} 3$ & 121.7 & $\mathrm{C} 11-\mathrm{C} 12-\mathrm{H} 12$ & 120.7 \\
\hline $\mathrm{C} 3-\mathrm{C} 4-\mathrm{C} 5$ & $123.3(3)$ & $\mathrm{C} 13-\mathrm{C} 12-\mathrm{H} 12$ & 120.7 \\
\hline $\mathrm{C} 3-\mathrm{C} 4-\mathrm{Br} 1$ & $117.6(3)$ & $\mathrm{C} 14-\mathrm{C} 13-\mathrm{C} 12$ & $121.8(3)$ \\
\hline $\mathrm{C} 5-\mathrm{C} 4-\mathrm{Br} 1$ & $119.1(3)$ & $\mathrm{C} 14-\mathrm{C} 13-\mathrm{H} 13$ & 119.1 \\
\hline $\mathrm{C} 6-\mathrm{C} 5-\mathrm{C} 4$ & $121.2(3)$ & $\mathrm{C} 12-\mathrm{C} 13-\mathrm{H} 13$ & 119.1 \\
\hline $\mathrm{C} 6-\mathrm{C} 5-\mathrm{H} 5$ & 119.4 & $\mathrm{C} 13-\mathrm{C} 14-\mathrm{C} 15$ & $118.2(3)$ \\
\hline $\mathrm{C} 4-\mathrm{C} 5-\mathrm{H} 5$ & 119.4 & $\mathrm{C} 13-\mathrm{C} 14-\mathrm{C} 17$ & $121.9(3)$ \\
\hline $\mathrm{C} 7-\mathrm{C} 6-\mathrm{C} 5$ & $114.6(3)$ & $\mathrm{C} 15-\mathrm{C} 14-\mathrm{C} 17$ & $120.0(3)$ \\
\hline $\mathrm{C} 7-\mathrm{C} 6-\mathrm{C} 9$ & $122.1(3)$ & $\mathrm{C} 16-\mathrm{C} 15-\mathrm{C} 14$ & $121.2(3)$ \\
\hline $\mathrm{C} 5-\mathrm{C} 6-\mathrm{C} 9$ & $123.3(3)$ & $\mathrm{C} 16-\mathrm{C} 15-\mathrm{H} 15$ & 119.4 \\
\hline $\mathrm{C} 6-\mathrm{C} 7-\mathrm{O} 1$ & $124.7(3)$ & $\mathrm{C} 14-\mathrm{C} 15-\mathrm{H} 15$ & 119.4 \\
\hline $\mathrm{C} 6-\mathrm{C} 7-\mathrm{C} 2$ & $125.3(3)$ & $\mathrm{C} 15-\mathrm{C} 16-\mathrm{C} 11$ & $119.3(3)$ \\
\hline $\mathrm{O} 1-\mathrm{C} 7-\mathrm{C} 2$ & $110.0(3)$ & $\mathrm{C} 15-\mathrm{C} 16-\mathrm{H} 16$ & 120.3 \\
\hline $\mathrm{C} 1-\mathrm{C} 8-\mathrm{O} 1$ & $110.8(3)$ & $\mathrm{C} 11-\mathrm{C} 16-\mathrm{H} 16$ & 120.3 \\
\hline $\mathrm{C} 1-\mathrm{C} 8-\mathrm{C} 10$ & $133.4(3)$ & $\mathrm{C} 14-\mathrm{C} 17-\mathrm{H} 17 \mathrm{~A}$ & 109.5 \\
\hline $\mathrm{O} 1-\mathrm{C} 8-\mathrm{C} 10$ & $115.8(3)$ & C14-C17-H17B & 109.5 \\
\hline $\mathrm{C} 6-\mathrm{C} 9-\mathrm{H} 9 \mathrm{~A}$ & 109.5 & $\mathrm{H} 17 \mathrm{~A}-\mathrm{C} 17-\mathrm{H} 17 \mathrm{~B}$ & 109.5 \\
\hline C6-C9-H9B & 109.5 & $\mathrm{C} 14-\mathrm{C} 17-\mathrm{H} 17 \mathrm{C}$ & 109.5 \\
\hline $\mathrm{H} 9 \mathrm{~A}-\mathrm{C} 9-\mathrm{H} 9 \mathrm{~B}$ & 109.5 & $\mathrm{H} 17 \mathrm{~A}-\mathrm{C} 17-\mathrm{H} 17 \mathrm{C}$ & 109.5 \\
\hline $\mathrm{C} 6-\mathrm{C} 9-\mathrm{H} 9 \mathrm{C}$ & 109.5 & $\mathrm{H} 17 \mathrm{~B}-\mathrm{C} 17-\mathrm{H} 17 \mathrm{C}$ & 109.5 \\
\hline $\mathrm{O} 2-\mathrm{S} 1-\mathrm{C} 1-\mathrm{C} 8$ & $-139.0(3)$ & $\mathrm{C} 1-\mathrm{C} 2-\mathrm{C} 7-\mathrm{C} 6$ & $-179.1(3)$ \\
\hline
\end{tabular}




$\begin{array}{llll}\mathrm{C} 11-\mathrm{S} 1-\mathrm{C} 1-\mathrm{C} 8 & 111.1(3) & \mathrm{C} 3-\mathrm{C} 2-\mathrm{C} 7-\mathrm{O} 1 & 179.8(3) \\ \mathrm{O} 2-\mathrm{S} 1-\mathrm{C} 1-\mathrm{C} 2 & 37.5(3) & \mathrm{C} 1-\mathrm{C} 2-\mathrm{C} 7-\mathrm{O} 1 & -0.1(3) \\ \mathrm{C} 11-\mathrm{S} 1-\mathrm{C} 1-\mathrm{C} 2 & -72.4(3) & \mathrm{C} 2-\mathrm{C} 1-\mathrm{C} 8-\mathrm{O} 1 & 0.9(3) \\ \mathrm{C} 8-\mathrm{C} 1-\mathrm{C} 2-\mathrm{C} 3 & 179.7(3) & \mathrm{S} 1-\mathrm{C} 1-\mathrm{C} 8-\mathrm{O} 1 & 177.9(2) \\ \mathrm{S} 1-\mathrm{C} 1-\mathrm{C} 2-\mathrm{C} 3 & 2.7(5) & \mathrm{C} 2-\mathrm{C} 1-\mathrm{C} 8-\mathrm{C} 10 & 178.6(3) \\ \mathrm{C} 8-\mathrm{C} 1-\mathrm{C} 2-\mathrm{C} 7 & -0.4(3) & \mathrm{S} 1-\mathrm{C} 1-\mathrm{C} 8-\mathrm{C} 10 & -4.3(5) \\ \mathrm{S} 1-\mathrm{C} 1-\mathrm{C} 2-\mathrm{C} 7 & -177.4(2) & \mathrm{C} 7-\mathrm{O} 1-\mathrm{C} 8-\mathrm{C} 1 & -0.9(3) \\ \mathrm{C} 7-\mathrm{C} 2-\mathrm{C} 3-\mathrm{C} 4 & 0.3(4) & \mathrm{C} 7-\mathrm{O} 1-\mathrm{C} 8-\mathrm{C} 10 & -179.1(2) \\ \mathrm{C} 1-\mathrm{C} 2-\mathrm{C} 3-\mathrm{C} 4 & -179.8(3) & \mathrm{O} 2-\mathrm{S} 1-\mathrm{C} 11-\mathrm{C} 12 & 13.2(3) \\ \mathrm{C} 2-\mathrm{C} 3-\mathrm{C} 4-\mathrm{C} 5 & -1.1(5) & \mathrm{C} 1-\mathrm{S} 1-\mathrm{C} 11-\mathrm{C} 12 & 123.1(3) \\ \mathrm{C} 2-\mathrm{C} 3-\mathrm{C} 4-\mathrm{B} 1 & 178.7(2) & \mathrm{O} 2-\mathrm{S} 1-\mathrm{C} 11-\mathrm{C} 16 & -171.8(3) \\ \mathrm{C} 3-\mathrm{C} 4-\mathrm{C} 5-\mathrm{C} 6 & 0.9(5) & \mathrm{C} 1-\mathrm{S} 1-\mathrm{C} 11-\mathrm{C} 16 & -62.0(3) \\ \mathrm{Br} 1-\mathrm{C} 4-\mathrm{C} 5-\mathrm{C} 6 & -179.0(2) & \mathrm{C} 16-\mathrm{C} 11-\mathrm{C} 12-\mathrm{C} 13 & 3.8(5) \\ \mathrm{C} 4-\mathrm{C} 5-\mathrm{C} 6-\mathrm{C} 7 & 0.2(4) & \mathrm{S} 1-\mathrm{C} 11-\mathrm{C} 12-\mathrm{C} 13 & 178.6(2) \\ \mathrm{C} 4-\mathrm{C} 5-\mathrm{C} 6-\mathrm{C} 9 & -179.6(3) & \mathrm{C} 11-\mathrm{C} 12-\mathrm{C} 13-\mathrm{C} 14 & -1.2(5) \\ \mathrm{C} 5-\mathrm{C} 6-\mathrm{C} 7-\mathrm{O} 1 & -179.9(3) & \mathrm{C} 12-\mathrm{C} 13-\mathrm{C} 14-\mathrm{C} 15 & -1.3(5) \\ \mathrm{C} 9-\mathrm{C} 6-\mathrm{C} 7-\mathrm{O} 1 & -0.1(5) & \mathrm{C} 12-\mathrm{C} 13-\mathrm{C} 14-\mathrm{C} 17 & 178.5(3) \\ \mathrm{C} 5-\mathrm{C} 6-\mathrm{C} 7-\mathrm{C} 2 & -1.1(5) & \mathrm{C} 13-\mathrm{C} 14-\mathrm{C} 15-\mathrm{C} 16 & 1.4(5) \\ \mathrm{C} 9-\mathrm{C} 6-\mathrm{C} 7-\mathrm{C} 2 & 178.7(3) & \mathrm{C} 17-\mathrm{C} 14-\mathrm{C} 15-\mathrm{C} 16 & -178.5(3) \\ \mathrm{C} 8-\mathrm{O} 1-\mathrm{C} 7-\mathrm{C} 6 & 179.6(3) & \mathrm{C} 14-\mathrm{C} 15-\mathrm{C} 16-\mathrm{C} 11 & 1.1(5) \\ \mathrm{C} 8-\mathrm{O} 1-\mathrm{C} 7-\mathrm{C} 2 & 0.6(3) & \mathrm{C} 12-\mathrm{C} 11-\mathrm{C} 16-\mathrm{C} 15 & -3.7(5) \\ \mathrm{C} 3-\mathrm{C} 2-\mathrm{C} 7-\mathrm{C} 6 & 0.8(5) & \mathrm{S} 1-\mathrm{C} 11-\mathrm{C} 16-\mathrm{C} 15 & -178.6(3) \\ & & & \end{array}$

Hydrogen-bond geometry $\left(A,{ }^{\circ}\right)$

$\mathrm{Cg}$ is the centroid of the C11-C16 4-methylphenyl ring.

\begin{tabular}{lllll}
\hline$D-\mathrm{H} \cdots A$ & $D-\mathrm{H}$ & $\mathrm{H} \cdots A$ & $D \cdots A$ & $D-\mathrm{H} \cdots A$ \\
\hline $\mathrm{C} 12-\mathrm{H} 12 \cdots \mathrm{O} 2^{\mathrm{i}}$ & 0.95 & 2.58 & $3.338(4)$ & 137 \\
$\mathrm{C} 17-\mathrm{H} 17 C \cdots C g^{\mathrm{ii}}$ & 0.98 & 2.77 & $3.739(4)$ & 169
\end{tabular}

Symmetry codes: (i) $-x+2,-y+1,-z+1$; (ii) $-x+1,-y+2,-z+1$. 